\title{
Confinement in 4-dimensional non-Abelian gauge theory
}

\section{E. Terry Tomboulis ${ }^{*+}$}

Univ. of California, Los Angeles

E-mail: tombouli@physics.ucla.edu

\begin{abstract}
A framework is developed in which approximate but explicitly computable renormalization group (RG) decimations provide upper and lower bounds at every scale for specifically chosen quantities such as partition functions. By interpolating between these bounds representations of the exact quantities are obtained at progressively longer scales (coarser lattices). This allows one to consider the behavior of order parameters. In the case of the SU(2) gauge theory RG flow to the confining strongly coupled regime is obtained for any initial coupling; whereas for the $\mathrm{U}(1)$ theory a fixed point is reached for small initial coupling. Other applications to the emergence of mass gap and the sufficiency of center confinement in SU(2) are also considered.
\end{abstract}

8th Conference Quark Confinement and the Hadron Spectrum

September 1-6 2008

Mainz, Germany

\footnotetext{
* Speaker.

${ }^{\dagger}$ Research supported by NSF-PHY-0555693
} 


\section{Introduction}

4-dimensional $S U(N)$ gauge theory at $T=0$ is known to be in a single confinement phase for $0<\beta<\infty$. At the same time the theory exhibits striking change in behavior over different length scales: passage from a short distance perturbative ordered regime to a long distance nonperturbative confining disordered regime. This behavior is quite analogous to that seen in another well-known case, the transition from laminar to turbulent flow in hydrodynamics. Such multi-scale problems are hard to treat. A natural framework is provided by a Renormalization Group (RG) block-spinning procedure tracing the evolution of the system over successive scales. The ultimate goal would be the construction of an exact block spinning procedure generating a trajectory converging to the Wilsonian renormalized trajectory emanating from the critical point and off the critical surface. Ideally, one would like to construct the action along the renormalized trajectory itself, the so-called 'perfect action', which would then allow one to compute any observable at any scale without contamination from irrelevant directions, hence no discretization effects. Despite many efforts, this lattice gauge theorist's Nirvana has not yet been reached.

A more modest but still sufficiently general framework is the proposal [1] to employ approximate, but easily explicitly computable RG decimation transformations that can provide bounds on specific, judicially chosen quantities. Examples of such quantities are partition functions (free energies) and/or their ratios. The strategy then is to consider only such quantities. This eschews the technically much more complicated construction of a general RG blocking action suitable for any observable. Using the bounds one is able constrain the corresponding exact quantities, and by then interpolating between the bounds obtain representations of the exact quantities under the RG decimation evolution. Such representations of the chosen quantities can serve as order parameters or provide other useful information which is hard to obtain by direct derivation from first principles by other means. In the following we develop this program in the case of 4-dimensional gauge theories on the lattice.

\section{Partition Function on progressively coarser lattices (longer scales)}

We employ standard lattice gauge theory notations, with $U$ denoting general group elements, $U_{b}$ bond variables, $U_{p}=\prod_{b \in \partial p} U_{b}$, etc. We start with some plaquette action on lattice $\Lambda$ of spacing $a$, for example the fundamental representation Wilson action:

$$
A_{p}(U)=\frac{\beta}{2} \chi_{1 / 2}\left(U_{p}\right)
$$

The character expansion of the exponential of plaquette action is then given by

$$
e^{A_{p}(U)}=\sum_{j} d_{j} F_{j}(\beta, a) \chi_{j}(U)=F_{0}\left[1+\sum_{j \neq 0} d_{j} c_{j}(\beta) \chi_{j}(U)\right]
$$

where $j$ labels representations. Thus, for $S U(2)$, our primary focus here, $j=0, \frac{1}{2}, 1, \frac{3}{2}, \ldots, \quad d_{j}=$ $(2 j+1)$. It is convenient to work in terms of the normalized coefficients $c_{j}(\beta)=F_{j}(\beta) / F_{0}(\beta)$. The (normalized) partition function (PF) on lattice $\Lambda$ is then

$$
Z_{\Lambda}(\beta)=\int d U_{\Lambda} \prod_{p}\left[1+\sum_{j \neq 0} d_{j} c_{j}(\beta) \chi_{j}(U)\right] \equiv Z_{\Lambda}\left(\left\{c_{j}(\beta)\right\}\right)
$$


For any reflection positive action one has $F_{j} \geq 0$, hence $1 \geq c_{j} \geq 0$ for all $j$.

$R G$ decimation We next introduce decimations to coarser lattices. We employ in particular transformations of the 'potential moving' type [2].

The RG decimation process can be summarized as a set of decimation rules for each successive step:

$$
\begin{aligned}
& a \rightarrow b a \rightarrow b^{2} a \rightarrow \cdots \rightarrow b^{n} a \\
& \Lambda \rightarrow \Lambda^{(1)} \rightarrow \Lambda^{(2)} \rightarrow \cdots \rightarrow \Lambda^{(n)}
\end{aligned}
$$

from lattice $\Lambda^{(m)}$ of spacing $b^{m} a$ to lattice $\Lambda^{(m+1)}$ of spacing $b^{m+1} a$, starting with the original lattice $\Lambda=\Lambda^{(0)}$. The rules give explicit expressions for the computation of the character expansion coefficients at the $m+1$-th step given those of the $m$-th step:

$$
\begin{aligned}
F_{0}(m) & =F_{0}\left(\zeta, b,\left\{c_{i}(m-1)\right\}\right) \\
c_{j}(m) & =c_{j}\left(\zeta, r, b,\left\{c_{i}(m-1)\right\}\right)
\end{aligned}
$$

The rule involves parameters $\zeta, r$ which control the amount by which the interactions of the remaining plaquettes after a decimation step are 'renormalized' to compensate for the ones that were removed. Such decimation parameters can, in general, be chosen differently at each decimation step. The explicit form of the recursion rules (2.4) - (2.5) need not be given here. It is only important to note that they are given by explicit algebraic recursion relations that can be computed to any desired accuracy.

Corresponding to (2.4) - (2.5) the PF undergoes the transformation

$$
Z_{\Lambda^{(m-1)}}\left(\left\{c_{j}(m-1)\right\}\right) \rightarrow F_{0}(m)^{\left|\Lambda^{(m)}\right|} Z_{\Lambda^{(m)}}\left(\left\{c_{j}(m)\right\}\right)
$$

There is a bulk free energy contribution resulting from the block-spinning $b^{(m-1)} a \rightarrow b^{m} a$ separated out from the PF $Z_{\Lambda^{(m)}}$ on the new lattice $\Lambda^{(m)}$. The important feature of the type of decimations used here is that, after such a decimation step, the resulting action $A_{p}(m)$ in $Z_{\Lambda^{(m)}}$ defined by

$$
\exp A_{p}(m)=\left[1+\sum_{j \neq 0} d_{j} c_{j}(m) \chi_{j}(U)\right]
$$

retains the original one-plaquette form but will, in general, contain all representations:

$$
A_{p}(m)=\sum_{j} \beta_{j}(m) \chi_{j}(U)
$$

Also, both positive and negative effective couplings $\beta_{j}(m)$ will occur. If, however, the parameter $\zeta=$ interger, all $c_{j}(m) \geq 0$. The resulting $\operatorname{PF} Z_{\Lambda^{(m)}}\left(\left\{c_{j}(m)\right\}\right)$ then retains the original form (2.3) and again defines a reflection positive measure on $\Lambda^{(m)}$.

Upper and lower bounds Consider the $\Lambda^{(m-1)} \rightarrow \Lambda^{(m)}$ decimation step with the following choices of decimation parameters: $c_{j}^{U}(m)$.

(a) $\zeta=b^{d-2}, \quad r=1-\varepsilon, \quad 0 \leq \varepsilon<1$; denote the resulting coefficients by $F_{0}^{U}(m)$ and

(b) $\zeta=1, \quad r=1$; denote resulting coefficients by $F_{0}^{L}(m)=1$ and $c_{j}^{L}(m)$. 
Then one has

$$
F_{0}^{L}(m)^{\left|\Lambda^{(m)}\right|} Z_{\Lambda^{(m)}}\left(\left\{c_{j}^{L}(m)\right\}\right)<Z_{\Lambda^{(m-1)}}<F_{0}^{U}(m)^{\left|\Lambda^{(m)}\right|} Z_{\Lambda^{(m)}}\left(\left\{c_{j}^{U}(m)\right\}\right) .
$$

Translation invariance, convexity of the free energy, and reflection positivity (positivity of character expansion coefficients) underlie the derivation of these bounds.

Interpolation It is now natural to interpolate between the upper and lower bounds in (2.9). Introducing a parameter $\alpha, 0 \leq \alpha \leq 1$, define interpolating coefficients $\tilde{c}_{j}(m, \alpha)$ and $\tilde{F}_{0}(m, \alpha)$ such that $\tilde{c}_{j}(m, 1)=c_{j}^{U}(m), \tilde{F}_{0}(m, 1)=F_{0}^{U}(m)$; and $\tilde{c}_{j}(m, 0)=c_{j}^{L}(m), \tilde{F}_{0}(m, 0)=F_{0}^{L}(m)$. But there is nothing unique about any one particular such interpolation. It is in fact expedient to more generally consider a family of such smooth interpolations parametrized by a parameter $t$ in some interval $\left(t_{a}, t_{b}\right)$. In fact, it suffices here to introduce $t$ as:

$$
\tilde{F}_{0}(m, \alpha, t)=\left\{\begin{array}{l}
F_{0}^{U}(m): \alpha=1 \\
F_{0}^{L}(m): \alpha=0
\end{array} \quad \tilde{c}_{j}(n, \alpha)=\left\{\begin{array}{l}
c_{j}^{U}(m): \alpha=1 \\
c_{j}^{L}(m): \alpha=0
\end{array}\right.\right.
$$

Example: $\tilde{F}_{0}(m, \alpha, t)=F_{0}^{U}(m)^{h(\alpha, t)}, F_{0}^{L}(m)=1$, with $h(0, t)=0, h(1, t)=1$, and, e.g., $h(\alpha, t)=$ $\exp \left\{-t \frac{1-\alpha}{\alpha}\right\}, 0<t_{a} \leq t \leq t_{b}<\infty$.

Define the corresponding interpolating Partition Function

$$
\tilde{Z}_{\Lambda^{(m)}}(\beta, m, \alpha, t, r)=\tilde{F}_{0}(m, \alpha, t)^{\left|\Lambda^{(m)}\right|} Z_{\Lambda^{(m)}}\left(\left\{\tilde{c}_{j}(m, \alpha, r)\right\}\right),
$$

where we now also made explicit the $r$ parameter dependence, cf. (2.4) - (2.5). Then the upperlower bounds statement (2.9) becomes

$$
\tilde{Z}_{\Lambda^{(m)}}(\beta, m, 0, t, r)<Z_{\Lambda^{(m-1)}}<\tilde{Z}_{\Lambda^{(m)}}(\beta, m, 1, t, r) .
$$

But then, by continuity, there exist a value

$$
0<\alpha=\alpha_{\Lambda}^{(m)}(t, r)<1
$$

such that

$$
\tilde{Z}_{\Lambda^{(m)}}\left(\beta, m, \alpha_{\Lambda}^{(m)}(t, r), t, r\right)=Z_{\Lambda^{(m-1)}}
$$

By construction there is parametrization invariance in (2.14) under shifts in t,r, i.e. $\alpha=\alpha_{\Lambda}^{(m)}(t, r)$ is the level surface fixed by the r.h.s. in (2.14). In particular, the derivatives $\partial \alpha_{\Lambda}^{(m)}(t, r) / \partial t$, $\partial \alpha_{\Lambda}^{(m)}(t, r) / \partial r$ are completely determined by (2.14). Also, the existence of the thermodynamic limit for the lattice system easily implies that

$$
\alpha_{\Lambda}^{(m)}(t, r)=\alpha^{(m)}(t, r)+\delta \alpha_{\Lambda}^{(m)}(t, r), \quad \delta \alpha_{\Lambda}^{(m)}(t, r) \rightarrow 0, \quad|\Lambda| \rightarrow \infty
$$

i.e. any lattice volume dependence in $\alpha_{\Lambda}^{(m)}(t, r)$ is weak, and goes away in the large volume limit.

So starting from original lattice, iterating this process of successive decimation and interpolation between upper and lower bounds one obtains an exact integral representation of the PF on 
progressively coarser lattices:

$$
\begin{aligned}
Z_{\Lambda}(\beta) & =Z_{\Lambda}\left(\left\{c_{j}(\beta)\right\}\right) \\
& =\tilde{F}_{0}\left(1, \alpha_{\Lambda}^{(1)}\left(t_{1}\right), t_{1}\right)^{\left|\Lambda^{(1)}\right|} Z_{\Lambda^{(1)}}\left(\left\{\tilde{c}_{j}\left(1, \alpha_{\Lambda}^{(1)}\left(t_{1}, r_{1}\right), r_{1}\right)\right\}\right) \\
& =\cdots \\
& =\left[\prod_{m=1}^{n} \tilde{F}_{0}\left(m, \alpha_{\Lambda}^{(m)}\left(t_{m}, r_{m}\right), t_{m}\right)^{|\Lambda| / b^{d m}}\right] Z_{\Lambda^{(n)}}\left(\left\{\tilde{c}_{j}\left(n, \alpha_{\Lambda}^{(n)}\left(t_{n}, r_{n}\right), r_{n}\right)\right\}\right) .
\end{aligned}
$$

The representation is given in terms of the accumulated bulk free energy contributions from the $a \rightarrow b^{n} a$ decimations, and a PF on the final lattice $\Lambda^{(n)}$ with a single plaquette effective action of the form (2.8). It should be noted that the action in this representation at different scales is, by construction, such as to reproduce the original exact free energy on the 1.h.s. It is not implied that the same action can give an exact representation for other quantities, though, for some, it may give a good approximation. This implements the program sketched in section 1.

\section{Partition function in the presence of external center flux}

Let $\mathscr{V}$ denote a coclosed (i.e. closed on the dual lattice) set of plaquettes completely winding around the periodic lattice in the $d-2$ directions normal to, say, the $x^{1}, x^{2}$ directions. Shift the plaquette action (2.1) by a a non-trivial element $\tau$ ('twist') of the group center on every plaquette in $\mathscr{V}$. For $S U(2)$ this amounts to flipping the sign: $\tau=-1 \in Z(2)$. Let $Z_{\Lambda}^{(-)}$denote the PF with twisted action on $\mathscr{V}$.

The twist amounts to a discontinuous (singular) gauge transformation on the configurations in the partition function which is multivalued in the center - so, for $S U(N)$, it is single-valued in $S U(N) / Z(N)$; i.e. it introduces a $\pi_{1}(S U(N) / Z(N))=Z(N)$ vortex. The set $\mathscr{V}$ represents the topological obstruction to having single-valuedness everywhere. It is easily shown that, as indicated by the notation, $Z_{\Lambda}^{(-)}$does not depend on the shape or location of $\mathscr{V}$, but only the presence of the center vortex flux rendered topologically stable by being wrapped around the lattice (torus) in the chosen directions.

Such twisted partition functions can be used for defining order parameters (section 4). The above development can now be carried through also in the presence of the twist. It is more convenient to actually work with the quantity $Z_{\Lambda}^{+}=\left(Z_{\Lambda}+Z_{\Lambda}^{(-)}\right) / 2$. Proceeding as in the previous section one obtains the analogous representation:

$$
\begin{aligned}
Z_{\Lambda}^{+} & =\left[\prod_{m=1}^{n} \tilde{F}_{0}\left(m, \alpha_{\Lambda}^{+(m)}\left(t_{m}, r_{m}\right), t_{m}\right)^{|\Lambda| / b^{d m}}\right] Z_{\Lambda^{(n)}}^{+}\left(\left\{\tilde{c}_{j}\left(n, \alpha_{\Lambda}^{+(n)}\left(t_{n}, r_{n}\right), r_{n}\right)\right\}\right) \\
Z_{\Lambda^{(n)}}^{+} & =\frac{1}{2}\left[Z_{\Lambda^{(n)}}\left(\left\{\tilde{c}_{j}\left(n, \alpha_{\Lambda}^{+(n)}\left(t_{n}, r_{n}\right), r_{n}\right)\right\}\right)+Z_{\Lambda^{(n)}}^{(-)}\left(\left\{\tilde{c}_{j}\left(n, \alpha_{\Lambda}^{+(n)}\left(t_{n}, r_{n}\right), r_{n}\right)\right\}\right)\right],
\end{aligned}
$$

where, again,

$$
\alpha_{\Lambda}^{+(m)}(t, r)=\alpha^{(m)}(t, r)+\delta \alpha_{\Lambda}^{+(m)}(t, r), \quad \delta \alpha_{\Lambda}^{+(m)}(t, r) \rightarrow 0, \quad|\Lambda| \rightarrow \infty
$$

As seen from (3.1), the external flux presence does not affect the bulk free-energy contributions (trivial characters) that resulted from successive blockings. Also, it should be noted that, as indicated by the notation, the $\alpha_{\Lambda}^{+(m)}(t, r)$ fixed at each successive step $m=1, \ldots, n$ in this representation 
are a priori distinct from the $\alpha_{\Lambda}^{(m)}(t)$ in the representation (2.16) for $Z_{\Lambda}(\beta)$. This is because they are fixed by an independent procedure involving a distinct quantity. It is easily seen, however, that any discrepancies between $\alpha_{\Lambda}^{+(m)}(t)$ and $\alpha_{\Lambda}^{(m)}(t)$ can only occur in the lattice-size dependent parts $\delta \alpha_{\Lambda}^{+(m)}(t), \delta \alpha_{\Lambda}^{(m)}(t)$. This assumes that the same family of interpolations is used in (2.16) and (3.1). In general, one may of course make different choices of interpolation in the two cases.

\section{Order parameters}

The vortex free-energy order parameter is defined as the ratio of the twisted to the untwisted PF:

$$
\exp \left(-F_{\Lambda}(\beta)\right)=\frac{Z_{\Lambda}^{(-)}}{Z_{\Lambda}} .
$$

Its behavior serves to characterize the various possible phases [3]. With $L_{\mu}$ denoting the length of the lattice in the $\mu$-direction, let $A=L_{1} L_{2}$ and $L=L_{3} \cdots L_{d}$. Then

$$
\begin{aligned}
& \left.F_{\Lambda}(\beta)\right) \sim \text { const } L \quad \Longleftrightarrow \quad \text { Higgs } \\
& \left.F_{\Lambda}(\beta)\right) \sim \text { const } L / A \quad \Longleftrightarrow \quad \text { Coulomb } \\
& \left.F_{\Lambda}(\beta)\right) \sim \text { const } L \exp (-\rho A) \quad \Longleftrightarrow \quad \text { Confinement }
\end{aligned}
$$

One may now represent (4.1), or equivalently, the quantity $Z_{\Lambda}^{+} / Z_{\Lambda}$, on successively decimated lattices by inserting our exact representations (3.1) and (2.16) in the numerator and denominator. Now, according to (2.15), (3.3), the $\alpha_{\Lambda}^{+(m)}(t, r)$ and $\alpha_{\Lambda}^{(m)}(t, r)$ in these representations differ only by quantities $\delta \alpha_{\Lambda}^{+(m)}(t, r), \delta \alpha_{\Lambda}^{(m)}(t, r)$ which vanish as $|\Lambda| \rightarrow \infty$. Still, to be able to use these representations to extract the behavior of (4.1) sufficiently accurately, one needs to account for these small discrepancies. This can be done in various ways by utilizing the (independent) parametrization invariance present in (3.1) and (2.16). One way proceeds in two steps as follows.

First, one may use the independent invariance under the parametrizations shifts in the representations in numerator and denominator to explicitly cancel the bulk free energy pieces generated at each decimation step. After the first decimation step one has

$$
\frac{Z_{\Lambda}^{+}}{Z_{\Lambda}}=\frac{\tilde{F}_{0}\left(1, \alpha_{\Lambda}^{+(1)}\left(t_{1}^{+}, r_{1}\right), t_{1}^{+}\right)^{\left|\Lambda^{(1)}\right|}}{\tilde{F}_{0}\left(1, \alpha_{\Lambda}^{(1)}\left(t_{1}, r_{1}\right), t_{1}\right)^{\left|\Lambda^{(1)}\right|}} \frac{Z_{\Lambda^{(1)}}^{+}\left(\left\{\tilde{c}_{j}\left(1, \alpha_{\Lambda}^{+(1)}\left(t_{1}^{+}, r_{1}\right)\right)\right\}\right)}{Z_{\Lambda^{(1)}}\left(\left\{\tilde{c}_{j}\left(1, \alpha_{\Lambda}^{(1)}\left(t_{1}, r_{1}\right)\right)\right\}\right)}
$$

with independent parameters $t_{1}, t_{1}^{+}$. Starting from some common value $t_{1}=t_{1}^{+}$, one may shift $t_{1}$ to a new nearby value such that

$$
\tilde{F}_{0}\left(1, \alpha_{\Lambda}^{(1)}\left(t_{1}, r_{1}\right), t_{1}\right)=\tilde{F}_{0}\left(1, \alpha_{\Lambda}^{+(1)}\left(t_{1}^{+}, r_{1}\right), t_{1}^{+}\right)
$$

Proceeding in this way in $n-1$ successive decimation steps one has:

$$
\frac{Z_{\Lambda}^{+}}{Z_{\Lambda}}=\frac{Z_{\Lambda^{(1)}}^{+}\left(\left\{\tilde{c}_{j}\left(1, \alpha_{\Lambda}^{+(1)}\left(t_{1}^{+}, r_{1}\right)\right)\right\}\right)}{Z_{\Lambda^{(1)}}\left(\left\{\tilde{c}_{j}\left(1, \alpha_{\Lambda}^{(1)}\left(t_{1}, r_{1}\right)\right)\right\}\right)}
$$




$$
\begin{aligned}
& =\frac{\tilde{F}_{0}\left(2, \alpha_{\Lambda}^{+(2)}\left(t_{2}^{+}, r_{2}\right), t_{2}^{+}\right)^{\left|\Lambda^{(2)}\right|} \frac{Z_{\Lambda^{(2)}}^{+}\left(\left\{\tilde{c}_{j}\left(2, \alpha_{\Lambda}^{+(2)}\left(t_{2}^{+}, r_{2}\right)\right)\right\}\right)}{\tilde{F}_{0}\left(2, \alpha_{\Lambda}^{(2)}\left(t_{2}, r_{2}\right), t_{2}\right)^{\left|\Lambda^{(2)}\right|}}=\cdots}{Z_{\Lambda^{(2)}}\left(\left\{\tilde{c}_{j}\left(2, \alpha_{\Lambda}^{(2)}\left(t_{2}, r_{2}\right)\right)\right\}\right)}=\cdots \\
& =\frac{Z_{\Lambda^{(n-1)}}^{+}\left(\left\{\tilde{c}_{j}\left(n-1, \alpha_{\Lambda}^{+(n-1)}\left(t_{n-1}^{+}, r_{n-1}\right), r_{n-1}\right)\right\}\right)}{Z_{\Lambda^{(n-1)}}\left(\left\{\tilde{c}_{j}\left(n-1, \alpha_{\Lambda}^{(n-1)}\left(t_{n-1}, r_{n-1}\right), r_{n-1}\right)\right\}\right)}
\end{aligned}
$$

This process is possible provided $\alpha^{(m)}(t) \leq 1-\delta$ for some lattice-size independent $\delta>0$, which can be shown to ensure that $\partial \alpha^{(m)} / \partial t>\eta>0$ for some lattice-size independent $\eta$. A sufficient condition for this is to set

$$
r_{m}=1-\frac{\varepsilon}{1+\beta_{\mathrm{eff}}(m-1)},
$$

where

$$
\beta_{\mathrm{eff}}(m)=-\frac{\partial^{2}}{\partial \theta^{2}} \ln \left[1+\sum_{j \neq 0} d_{j} \tilde{c}_{j}(m) \chi_{j}(\theta)\right]_{\theta=0}
$$

for some small $\varepsilon \sim 10^{-6}$.

Having arrived at (4.4) with $n$ large enough, perform next one more decimation step to get

$$
\frac{Z_{\Lambda}^{+}}{Z_{\Lambda}}=\frac{Z_{\Lambda^{(n-1)}}^{+}}{Z_{\Lambda^{(n-1)}}}=\frac{\tilde{F}_{0}\left(n, \alpha_{\Lambda}^{+(n)}\left(t_{n}, r_{n}\right), t_{n}\right)^{\left|\Lambda^{(n)}\right|}}{\tilde{F}_{0}\left(n, \alpha_{\Lambda}^{(n)}\left(t_{n}, r_{n}\right), t_{n}\right)^{\left|\Lambda^{(n)}\right|}} \frac{Z_{\Lambda^{(n)}}^{+}\left(\left\{\tilde{c}_{j}\left(n, \alpha_{\Lambda}^{+(n)}\left(t_{n}, r_{n}\right)\right)\right\}\right)}{Z_{\Lambda^{(n)}}\left(\left\{\tilde{c}_{j}\left(n, \alpha_{\Lambda}^{+(n)}\left(t_{n}, r_{n}\right)\right)\right\}\right)} .
$$

Now seek a value $t_{n}=t_{\Lambda}^{*}\left(r_{n}\right)$ such that

$$
\alpha_{\Lambda}^{(n)}\left(t_{\Lambda}^{*}, r_{n}\right)=\alpha_{\Lambda}^{+(n)}\left(t_{\Lambda}^{*}, r_{n}\right) \equiv \alpha_{\Lambda}^{*(n)}\left(r_{n}\right) .
$$

This is equivalent to seeking a solution $(\alpha, t)$ (for given $r$ ) to the system of two simultaneous nonlinear equations

$$
\begin{aligned}
& -\tilde{F}_{0}(n, \alpha, t)-\frac{1}{\left|\Lambda^{(n)}\right|} \ln Z_{\Lambda^{(n)}}^{+}(\alpha, t)+\frac{b^{d}}{\left|\Lambda^{(n-1)}\right|} \ln Z_{\Lambda^{(n-1)}}^{+}=0 \\
& -\tilde{F}_{0}(n, \alpha, t)-\frac{1}{\left|\Lambda^{(n)}\right|} \ln Z_{\Lambda^{(n)}}(\alpha, t)+\frac{b^{d}}{\left|\Lambda^{(n-1)}\right|} \ln Z_{\Lambda^{(n-1)}}=0 .
\end{aligned}
$$

Let us first see heuristically why, in the case of $S U(2)$, we know that this system indeed has a solution. Computation of the flow of the upper bound coefficients $c_{j}^{U}(m)$, i.e. evaluation of the flow at $\alpha=1$, leads to the strong coupling confining regime with a string tension which is lower compared to that obtained, by simulation, in the exact theory. This is in fact evident from the direction of flow set from weak coupling by the beta functions for the exact versus the $\alpha=1$ case (with $\varepsilon=0$ or $\varepsilon>0$ ). The limit $\alpha=0$, on the other hand, corresponds to a superconfining limit. In other words, the following relation is seen numerically to hold

$$
\left[\frac{Z_{\Lambda^{(n)}}^{(-)}(\alpha)}{Z_{\Lambda^{(n)}}(\alpha)}\right]_{\alpha=0}>\frac{Z_{\Lambda}^{(-)}}{Z_{\Lambda}}>\left[\frac{Z_{\Lambda^{(n)}}^{-}(\alpha)}{Z_{\Lambda^{(n)}}(\alpha)}\right]_{\alpha=1} .
$$

It follows that there is an $\alpha^{*}$ in between such that $Z_{\Lambda}^{(-)} / Z_{\Lambda}=Z_{\Lambda^{(n)}}^{(-)}\left(\alpha^{*}\right) / Z_{\Lambda^{(n)}}\left(\alpha^{*}\right)$, which, by (4.7), is equivalent to a solution of (4.9). 
To solve the system (4.9) from first principles we employ a technique of monotone iteration. Let

$$
x=(t, \alpha) \in D \subset \mathbf{R}^{2}, \quad F(x)=\left(f_{1}, f_{2}\right): \mathbf{R}^{2} \rightarrow \mathbf{R}^{2} .
$$

We seek solution to: $F(x)=0$.

Consider the sequence of points $x_{k}\left(\lambda_{0}\right)$ iteratively obtained from

$$
\begin{aligned}
d x_{k}(\lambda) / d \lambda & =-\left[1+\lambda F_{, x}\left(x_{k}\right)\right]^{-1} \cdot F\left(x_{k}\right) \\
x_{k}(0) & =x_{k-1}\left(\lambda_{0}\right), \quad x_{1}(0)=x_{0} \quad k=1,2, \ldots .
\end{aligned}
$$

Note that each function $x_{k}(\lambda)$ solving (4.10) is a solution to the homotopy

$$
\Psi\left(x_{k}, \lambda\right) \equiv\left(x_{k}-x_{k}(0)\right)+\lambda F\left(x_{k}\right)=0 .
$$

If $M$ is a matrix, by $M \geq 0$ we mean that all its elements are non-negative. One now has the following result:

If: $\quad$ (i) $F\left(x_{0}\right)<0 ; \quad$ (ii) $\left[1+\lambda F_{, x}\right]^{-1} \geq 0$ for $\lambda \in\left[0, \lambda_{0}\right]$ and all $x \in D$,

then the sequence of points obtained from (4.10) is monotonically increasing [4]. Hence, it will be convergent if it stays within $D$ for bounded $D$. This is assured if at least one component of $F$ is positive on $\partial D$. It is then converging to a solution $x^{*}=\left(t^{*}, \alpha^{*}\right)$ of $F(x)=0$.

The above conditions are satisfied in the case of the system (4.9) for $n$ large enough and appropriate choice of the class of parametrization functions $h$. This then provides a constructive proof of the existence of solutions to the system (4.9), or equivalently to (4.8). Thus, from (4.7), the end result is

$$
\frac{Z_{\Lambda}^{(-)}}{Z_{\Lambda}}=\frac{Z_{\Lambda^{(n)}}^{(-)}\left(\left\{\tilde{c}_{j}\left(n, \alpha_{\Lambda}^{*(n)}\right)\right\}\right)}{Z_{\Lambda^{(n)}}\left(\left\{\tilde{c}_{j}\left(n, \alpha_{\Lambda}^{*(n)}\right)\right\}\right)} .
$$

(4.12) is the desired result that allows one to compute the vortex free energy. Indeed, the coefficients in terms of which the PF's in (4.12) are given are bounded by the upper bound coefficients $c_{j}^{U}(n)$, which, by straightforward evaluation of the decimation rules, flow into the strong coupling regime:

$$
\tilde{c}_{j}\left(n, \alpha_{\Lambda}^{*(n)}\right) \leq c_{j}^{U}(n) \rightarrow 0 \quad \text { for } \quad n \rightarrow \infty .
$$

Hence one can compute the r.h.s. of the exact representation (4.12) in the convergent strong coupling expansion. There are several immediate consequences:

- Confining behavior is the result for any initial $\beta$ since $c_{j}^{U}(n) \rightarrow 0$ for $n \rightarrow \infty$ for any initial $\beta$.

- Fixing the resulting string tension $\kappa(\beta, n)$ implies a relation between $n$ and $\beta=2 / g^{2}$.

- Now zero coupling $g=0$ is a fixed point of the decimations. This implies that to reach any fixed value of the string tension (equivalently, some given value of $c_{j}^{U}(n)$ 's) requires $n \rightarrow \infty \quad \Longleftrightarrow \quad \beta \rightarrow \infty$. In other words one necessarily has

$$
g(a) \rightarrow 0 \quad \text { for } \quad a \rightarrow 0,
$$

i.e. asymptotic UV freedom, as an essentially qualitative feature of the decimation flow. 
Other groups $S U(3)$ : This is rather similar to $S U(2)$ except for the modifications due to the $Z(3)$ center. The important point is that upper and lower bounding flows are known to be qualitatively similar to the $S U(2)$ case. Therefore, the same general picture is expected.

$U(1)$ : The same development can be applied to $U(1)$. Here, however, one encounters a crucial difference. Starting from weak coupling (initial $\beta$ large enough), the flow under successive decimation with (4.5) hits a fixed point. The process leading to (4.4) thus never reaches the strong coupling regime. The need for $\varepsilon>0$ to reproduce the original system at each stage can actually be seen from the detailed analysis of the decimation flows at $\varepsilon=0$ [5].

Mass gap By known inequalities relating the vortex free energy to the Wilson loop [6], confining behavior for vortex free energy implies area law for the Wilson loop. It also implies the existence of mass gap. Consider the correlator of two Wilson lines located at points $x$ and $y$. If the length of the line is $L_{W}$, by a similar argument as in [6], confining vortex free energy gives

$$
\left\langle W_{x} W_{y}\right\rangle_{\Lambda}^{1 / L_{W}} \leq \exp [-\rho|x-y|]
$$

as $|\Lambda| \rightarrow \infty,|x-y| \rightarrow \infty$, i.e. exponential clustering of Wilson line correlators. Furthermore, since, by reflection positivity,

$$
\left\langle W_{x}\right\rangle_{\Lambda} \leq\left\langle W_{x} W_{y}\right\rangle^{1 / 2}
$$

(4.15) implies absence of 'magnetization' (vanishing of Wilson line expectation), i.e. absence of breaking of $Z(2)$ global symmetry. This, as it is well-known, is an alternative criterion for being in the confining phase.

Center confi nement It is an old result that "if the center confines, the whole group confines" [7]. This interesting sufficiency criterion, however, as originally derived, applies to the scale at which the theory is defined, i.e. lattice spacing (UV regulator). It is thus not useful in the physically interesting cases of weak short distance coupling. E.g. for $S U(N)$, the center $Z(N)$ is non-confining at weak coupling.

Such a result, however, would be useful if obtained at an effective action level at the appropriate scale. This is in fact another old idea, a $Z(N)$ gauge theory as an effective theory for the $S U(N)$ theory at large distances [8].

It is now possible to derive the corresponding statement using our representations of the partition functions on coarser lattices: if the center confines on a sufficiently coarser lattice than the one on which the gauge theory is originally defined, the theory confines.

Now, the PF of any $Z(N)$ lattice gauge theory can be exactly rewritten as the PF of $(d-2)$ dimensional objects conserved $\bmod N$, i.e. $Z(N)$ vortices. Thus the result implies that percolation of sufficiently 'fat' center vortices is sufficient for confinement in $S U(N)$ gauge theory $(N=2,3)$.

\section{Summary}

A framework was developed that utilizes approximate but explicitly computable RG decimation transformations to constrain the behavior of the exact theory. This has many potential applications. We applied it to the derivation of exact integral representations of the PF with or without external center flux on successively coarser lattices, i.e. longer scales. These were then used to 
examine order parameters, in particular the vortex free energy which is built out of such partition functions. Applied to the case of the $S U(2)$ gauge theory, confinement at $T=0$ emerges for any initial coupling once the approximate bounding decimations possess this property. The extension to $S U(3)$ should be straightforward since the same qualitative flow under the bounding decimations is known to occur. In contrast, application to $U(1)$ leads to a fixed point preventing flow from the weak coupling to the strong coupling regime. Some other applications, such as the existence of mass gap and the sufficiency of center confinement in the confining phase of $S U(2)$ were also briefly considered. Application of the formalism to 2-dimensional spin models, in particular the Heisenberg SU(2) model, and combining with Monte Carlo RG, are among several other possible applications not considered here. It would be very desirable to develop usable RG decimation schemes for interacting fermions that could then be used in a similar framework. No such schemes, however, are presently available.

\section{References}

[1] E.T. Tomboulis, Confinement for all values of the coupling in four dimensional $S U(2)$ gauge theory, arXiv:0707.2179.

[2] A.A. Migdal, Recursion equations in gauge theories, Sov. Phys. JETP, 42, 413 (1976); L.P. Kadanoff, Notes on Migdal's recursion formulas, Ann. Phys. (N.Y.) 91, 226 (1975).

[3] G. 't Hooft, A property of electric and magnetic flux in non-Abelian gauge theories, Nucl. Phys. B153, 141 (1979); L.G. Yaffe, Confinement in SU(N) lattice gauge theories, Phys. Rev. D 21, 1574 (1980).

[4] G. Meyer, On solving nonlinear equations with a one-parameter operator imbedding, SIAM J. Numer. Anal., 5739 (1968).

[5] J.V. José, L.P. Kadanoff, S.Kirkpatrick and D.R. Nelson, Renormalization, vortices, and symmetry-breaking perturbations in the 2-dimensional planar model, Phys. Rev. B16, 1217 (1977).

[6] E.T. Tomboulis and L.G. Yaffe, Finite Temperature SU(2) Lattice Gauge Theory, Commun. Math. Phys. 100, 313 (1985): T.G. Kovács and E.T. Tomboulis, Vortex waistlines and long range fluctuations, Phys. Rev. D 65, 074501 (2002) [arXiv:hep-lat/0108017].

[7] G. Mack and V.B. Petkova, Comparison of Lattice gauge Theories with Gauge Groups Z(2) and SU(2), Ann. Phys. (N.Y.) 123, 442 (1979); J. Frölich, Confinement in Z(n) lattice gauge theories implies confinement in SU(n) lattice Higgs theories, Phys. Lett. 83B, 195 (1979).

[8] G. Mack, Predictions of a theory of quark confinement, Phys. Rev. Lett. 45, 1378 (80), E.T. Tomboulis, 't Hooft loop in SU(2) lattice gauge theories, Phys. Rev. D 23, 2371 (1981). 